\title{
Inserção no mercado de trabalho de egressos de um curso de Odontologia do Piauí
}

\author{
Ana Flávia Andrade Sérgio*; Cacilda Castelo Branco Lima**; Patrícia Ferreira de Sousa Viana** \\ * Graduada em Odontologia pela Universidade Federal do \\ Piauí \\ ** Professora, Departamento de Patologia e Clínica \\ Odontológica, Universidade Federal do Piauí
}

Recebido em 09/04/2020. Aprovado em 27/07/2020.

\begin{abstract}
RESUMO
As transformações e dificuldades que permeiam o mercado de trabalho odontológico despertam questões sobre a inserção dos cirurgiões-dentistas e sua formação diante do exercício profissional. O objetivo deste trabalho foi avaliar a inserção de egressos do curso de Odontologia da Universidade Federal do Piauí no mercado de trabalho. Neste estudo transversal de caráter quantitativo, um questionário online autoaplicável foi enviado a 76 cirurgiões-dentistas formados entre 2017 e 2018 , abordando dados sociodemográficos, investimento em pós-graduação e ingresso no mercado de trabalho. Foi realizada análise descritiva dos dados. A taxa de resposta foi de $92,1 \%$. A maioria dos egressos $(88,6 \%)$ possuía ou cursava algum tipo de pós-graduação, principalmente nas áreas de Cirurgia, Endodontia e Ortodontia. Quanto ao ingresso no mercado, apenas 4,3\% nunca exerceram a profissão e $75,7 \%$ se inseriram em até 3 meses de formados. Os cirurgiões-dentistas estão divididos de forma equiparável entre os setores público e privado, possuem em sua maioria jornadas de até 40 horas $(75,4 \%)$ e renda mensal de até 5 salários mínimos (91,3\%). Apenas 11,4\% não estão satisfeitos com a profissão escolhida e $40 \%$ julgam o mercado como regular. Entre as dificuldades relatadas no início da profissão, as mais citadas foram baixa remuneração $(82,1 \%)$ e insegurança na prática clínica $(70,1 \%)$. Como sugestão para o curso de graduação, indicaram a abordagem de Administração, Marketing e Empreendedorismo. Observou-se que o mercado proporcionou oportunidades para uma inserção rápida, no entanto, com redução do exercício estritamente autônomo. Os egressos valorizam a pós-graduação e apesar das dificuldades, estão satisfeitos com a profissão.
\end{abstract}

Descritores: Mercado de Trabalho. Odontologia. Recursos Humanos em Odontologia. 


\section{INTRODUÇÃO}

O mercado de trabalho odontológico está inserido em um cenário dinâmico, determinado por diversos fatores, dos quais evidenciam-se os padrões epidemiológicos, o contexto cultural e econômico, o modelo de prestação de serviço e a oferta de mão de obra ${ }^{1}$. Observa-se um progressivo movimento de redução da prática privada autônoma estrita, com a popularização dos sistemas de Odontologia em grupo e expansão do trabalho no serviço público².

Até o final do século $\mathrm{XX}$, a profissão odontológica era predominantemente autônoma, baseada numa abordagem tecnicista, curativa e especializada ${ }^{3}$. A partir da década de 90 , o mercado de trabalho em saúde começava a apresentar uma flexibilização das formas de inserção dos trabalhadores no país, que trouxe modificações ao cenário odontológico até então predominantemente autônomo ${ }^{4}$.

$\mathrm{Na}$ esfera privada, a saúde suplementar começava a ganhar espaço ${ }^{4,5}$. Os convênios e credenciamentos tornaram-se intermediários na relação paciente-profissional, como estratégia de captação de pacientes ${ }^{4}$. No setor público, a inserção das equipes de saúde bucal no então Programa Saúde da Família, no início de 2001, expandiu o mercado de trabalho ${ }^{6}$ e ressignificou o papel dos cirurgiões-dentistas na saúde coletiva ${ }^{7}$.

Três anos mais tarde, a Política Nacional de Saúde Bucal - o Brasil Sorridente, expandiu as equipes de saúde bucal e organizou uma rede de referência e contrarreferência por meio dos Centros de Especialidades Odontológicas $(\mathrm{CEO})^{8}$. Desde então, o Sistema Único de Saúde (SUS) passou a ser um grande empregador para os cirurgiõesdentistas $^{9}$ - atualmente, são mais de 28 mil postos de trabalho no Brasil para CD na Estratégia Saúde da Família (ESF) ${ }^{10}$.

As mudanças nesse contexto evidenciaram a insuficiência da formação odontológica tradicional - com ênfase em práticas fragmentadas e atuação quase exclusiva no mercado privado, o que apontou para novos desafios ao ensino odontológico ${ }^{11}$. Como marco teórico-metodológico do processo de reforma da educação superior, foram lançadas, em 2002, as Diretrizes Curriculares Nacionais (DCN) para o curso de Odontologia. As DCN estabeleceram novas referências para currículo, com o intuito de aproximar o perfil do egresso às demandas sociais, em consonância com o SUS ${ }^{12}$.

Paralelamente, houve um aumento expressivo de cursos de graduação em Odontologia. De 2015 a 2018, o número de cursos passou de 241 para $385^{13,14}$. A maior oferta de vagas para graduação em Odontologia culminou no aumento da oferta de mão de obra, sem um planejamento na distribuição e na capacidade do mercado de trabalho para absorver tal incremento $^{15}$. O Brasil possui hoje mais de 339 mil cirurgiões-dentistas, com $52,7 \%$ destes concentrados na região Sudeste ${ }^{16}$. No Nordeste, além da concentração de CD nas capitais, observase ainda um déficit de profissionais no interior dos estados ${ }^{17}$. O reflexo disso é a saturação do mercado autônomo, que traz como consequências o aumento da competitividade, a precarização das condições de trabalho e desvalorização da profissão ${ }^{18,19}$.

O curso de Odontologia da Universidade Federal do Piauí (UFPI), motivado pelo Programa Nacional de Reorientação da Formação Profissional em Saúde (Pró-Saúde), em 2007, reformulou seu projeto pedagógico e sua matriz curricular em consonância com as DCN, com o intuito de aproximar o perfil do egresso às necessidades de um mercado de trabalho que se voltava para outros setores, especialmente o público, no âmbito do Sistema Único de Saúde ${ }^{20}$.

As dificuldades que permeiam o cenário atual do mercado de trabalho odontológico despertam questões sobre a inserção dos cirurgiões-dentistas, em especial no início de sua carreira profissional, quando ainda não se apresentam estabelecidos na profissão. Conhecer o perfil dos profissionais poderá nortear "mudanças 
na profissão, novas tendências e necessidades do mercado de trabalho, além de orientar futuras decisões dos gestores do curso" 21 .

Nesse contexto, o objetivo deste trabalho foi avaliar a inserção de egressos do curso de graduação em Odontologia da Universidade Federal do Piauí no mercado de trabalho, identificando o perfil, as escolhas de carreira e possíveis dificuldades enfrentadas.

\section{METODOLOGIA}

Trata-se de um estudo transversal, de caráter quantitativo. A população foi constituída pelos cirurgiões-dentistas egressos do curso de graduação em Odontologia da UFPI formados nos anos de 2017 e 2018, totalizando 80 indivíduos. Para o cálculo amostral, foi adotada a prevalência de $95,1 \%$ de inserção dos CD no mercado de trabalho $^{22}$, e aplicado um fator de correção para desenho de estudo de 1,8 para aumentar a precisão da amostra, com um intervalo de confiança de $95 \%$ e margem de erro de 5\%. Assim, o tamanho mínimo da amostra foi definido em 69 egressos, e com o acréscimo de $10 \%$ para compensar eventuais perdas, 76 indivíduos foram convidados a participar da pesquisa.

$\mathrm{O}$ instrumento de coleta consistiu em um questionário online autoaplicável, hospedado na plataforma Google-Forms ${ }^{\circledR}$ (Mountain View, CA, USA: Google LLC), composto de 20 questões (12 fechadas e 8 abertas). As variáveis abordadas foram: idade, sexo, modalidade e área de pósgraduação, tempo de ingresso no mercado, município e setor de atuação, tipo de vínculo, carga horária, remuneração, percepção sobre o mercado de trabalho, satisfação profissional, motivos para o não exercício da Odontologia, dificuldades enfrentadas no início do exercício profissional e sugestões ao curso de graduação.

Após estudo piloto com uma amostra de conveniência e ajuste do instrumento, procedeu-se à coleta de dados. Os egressos foram identificados na coordenação do curso e contatados via WhatsApp ${ }^{\circledR}$ (Menlo Park, CA, USA: WhatsApp Inc) em setembro de 2019, para envio do termo de consentimento livre e esclarecido e link de acesso ao questionário. Nos casos em que não houve retorno em até 7 dias, realizou-se outras duas tentativas de contato, com intervalo de 7 dias entre cada.

O processamento dos dados e análise estatística foi realizada no programa Statistical Package for the Social Sciences (SPSS ${ }^{\circledR}$ para Windows, versão 20.0, Armonk, NY, USA: IBM Corp). Foram realizadas a descrição de frequências absolutas e relativas das variáveis. Devido à grande diversidade das respostas de duas questões abertas, referentes aos municípios de atuação profissional e as sugestões para o curso de graduação, foi necessário agrupá-las em categorias, para a análise descritiva. Para a primeira questão, utilizou-se como critérios o tipo de região (metropolitana ou interior) e o estado (se no Piauí e/ou outro estado). Com relação as sugestões para o curso, as respostas foram categorizadas de acordo com as áreas do conhecimento sugeridas para melhorar a abordagem/aprofundamento e àquelas para serem implementas no curso.

Este estudo foi aprovado pelo Comitê de Ética em Pesquisa da UFPI (parecer 3.553.410/2019).

\section{RESULTADOS}

Dos 76 egressos convidados para a pesquisa, 70 responderam ao questionário (taxa de resposta de 92,1\%). Na tabela 1, estão descritas informações sobre o perfil dos participantes. A maioria era do sexo feminino $(68,6 \%)$, com idade entre 22 e 25 anos $(81,4 \%)$. Em relação à pós-graduação, 88,6\% dos egressos possuíam ou estavam cursando algum tipo, entre os quais destacaram-se especialização $(54,3 \%)$ e aperfeiçoamento $(42,8 \%)$. As áreas mais citadas foram Cirurgia, Endodontia e Ortodontia (tabela 1). 
Tabela 1. Perfil dos participantes do estudo

\begin{tabular}{lcc}
\hline \multicolumn{1}{c}{ Variáveis } & n & \% \\
\hline Sexo & 22 & 31,4 \\
Masculino & 48 & 68,6 \\
Feminino & & \\
Faixa etária (em anos) & 57 & 81,4 \\
$22-25$ & 13 & 18,6 \\
$26-31$ & & \\
Período de conclusão da graduação & 13 & 18,5 \\
2017.1 & 20 & 28,6 \\
2017.2 & 17 & 24,3 \\
2018.1 & 20 & 28,6 \\
2018.2 & & \\
Pós-graduação & 12 & 17,1 \\
Aperfeiçoamento & 20 & 28,6 \\
Especialização & 1 & 1,4 \\
Residência & 8 & 11,4 \\
Mestrado & 13 & 18,6 \\
Aperfeiçoamento e especialização & 3 & 4,3 \\
Aperfeiçoamento e mestrado & 3 & 4,3 \\
Especialização e mestrado & 1 & 1,4 \\
Aperfeiçoamento, especialização e mestrado & 1 & 1,4 \\
Aperfeiçoamento, especialização e residência & 8 & 11,4 \\
Não possui & & \\
Área da Pós-graduação* & 23 & 37,1 \\
Cirurgia & 19 & 30,6 \\
Endodontia & 2 & 3,2 \\
Periodontia & 15 & 24,2 \\
Ortodontia & 4 & 6,5 \\
Implantodontia & 6 & 9,7 \\
Saúde coletiva & 3 & 4,8 \\
Prótese & 1 & 1,6 \\
Radiologia & 1 & 1,6 \\
Pacientes especiais & 1 & 1,6 \\
Odontologia legal & 2 & 3,2 \\
Harmonização Orofacial & 12 & 19,4 \\
Clínica Odontológica & $\mathbf{7 0}$ & $\mathbf{1 0 0 , 0}$ \\
\hline Total & & \\
\hline & &
\end{tabular}

*Os participantes poderiam responder mais de uma área de pós-graduação e foram considerados os percentuais válidos.

De acordo com a tabela 2, $81,4 \%$ estavam inseridos no mercado. O tempo de inserção no mercado de trabalho foi de até 3 meses após a graduação para $75,7 \%$ dos egressos, sendo que $45,1 \%$ ingressaram em menos de 1 mês. Em relação à distribuição geográfica, os CD se estabeleceram profissionalmente de forma equiparável entre a região metropolitana de Teresina $(52,6 \%)$ e interior $(47,4 \%)$. Ressalta-se que $36,8 \%$ atuavam em outros estados - Maranhão, Ceará, Pará e Mato Grosso. Entre os 13 participantes que não mantinham vínculo 
empregatício na área da Odontologia $(18,6 \%)$, o principal motivo foi a dedicação à pós-graduação $(12,8 \%)$.
Apenas dois egressos relataram a busca por outra graduação, por frustração com a Odontologia $(2,9 \%)$ (tabela 2).

Tabela 2. Caracterização dos cirurgiões-dentistas quanto ao exercício profissional, região e Estado de atuação, motivos para o não exercício e tempo de ingresso no mercado

\begin{tabular}{lcc}
\hline \multicolumn{1}{c}{ Variáveis } & $\mathbf{n}$ & $\mathbf{\%}$ \\
\hline Exercício da profissão & & \\
Exerce atualmente & 57 & 81,4 \\
Já exerceu, mas não atualmente & 10 & 14,3 \\
Nunca exerceu a profissão & 3 & 4,3 \\
Tipo de Região* & & \\
$\quad$ Região Metropolitana de Teresina & 26 & 45,6 \\
Região Metropolitana de Teresina e interior & 4 & 7,0 \\
Interior & 27 & 47,4 \\
Estado* & 36 & 63,2 \\
Piauí & 4 & 7,0 \\
Piauí e outro estado & 17 & 29,8 \\
Outro estado & & \\
Motivos para o não exercício & 57 & 81,4 \\
Exerce atualmente & 9 & 12,8 \\
Dedicação à pós-graduação & 2 & 2,9 \\
Buscou outra graduação & 2 & 2,9 \\
Outro & & \\
Tempo de ingresso no mercado (meses) & 3 & 4,3 \\
Nunca exerceu a profissão & 32 & 45,7 \\
< 1 & 21 & 30,0 \\
1 - 3 & 8 & 11,4 \\
$4-6$ & 6 & 8,6 \\
\hline 6 & $\mathbf{7 0}$ & $\mathbf{1 0 0 , 0}$ \\
\hline Total
\end{tabular}

$*_{n}=57$ que corresponde aos cirurgiões-dentistas que exercem atualmente a profissão e foram considerados os percentuais válidos

Quanto aos setores de atuação, predominou o exercício mútuo nos setores público e privado $(38,6 \%)$. Na tabela 3, estão descritos o vínculo de trabalho, a carga horária, a renda mensal, remuneração ideal e classificação do salário, de acordo com o setor.

No setor público, o vínculo predominante foi o contrato com carteira assinada $(57,1 \%)$, seguido do trabalho sem contrato $(23,8 \%)$. No setor privado, a forma de atuação mais citada foi a prestação de serviços $(43,2 \%)$, e o exercício autônomo, a menos prevalente $(21,6 \%)$. Para $68,4 \%$, a carga horária correspondente foi entre 31 a mais de 40 horas semanais. A remuneração mensal variou entre 1 e 9 salários mínimos, e a maioria recebe até 5 salários mínimos $(91,3 \%)$. Os egressos classificaram seu salário como bom ou regular $(77,2 \%)$ e consideraram uma remuneração ideal na faixa de 3 a 10 salários mínimos $(82,5 \%)$ (tabela 3). 
Tabela 3. Vínculos de trabalho, carga horária semanal, renda mensal média, remuneração ideal e classificação do salário dos cirurgiões-dentistas que exercem a profissão, de acordo com o setor de atuação

\begin{tabular}{|c|c|c|c|c|}
\hline \multirow[b]{2}{*}{ Variáveis } & \multicolumn{4}{|c|}{ Setor } \\
\hline & $\begin{array}{c}\text { Público } \\
\text { n (\%) }\end{array}$ & $\begin{array}{c}\text { Privado } \\
\text { n (\%) }\end{array}$ & $\begin{array}{c}\text { Público e } \\
\text { Privado } \\
\text { n (\%) }\end{array}$ & $\begin{array}{l}\text { Total } \\
\text { n(\%) }\end{array}$ \\
\hline \multicolumn{5}{|l|}{ Vínculo Público } \\
\hline $\begin{array}{l}\text { Contrato com carteira } \\
\text { assinada }\end{array}$ & $13(65,0)$ & - & $11(50,0)$ & $24(57,1)$ \\
\hline $\begin{array}{l}\text { Contrato sem carteira } \\
\text { assinada }\end{array}$ & $2(10,0)$ & - & $3(13,6)$ & $5(11,9)$ \\
\hline Estatutário & $1(5,0)$ & - & $2(9,1)$ & $3(7,1)$ \\
\hline Sem contrato & $4(20,0)$ & - & $6(27,3)$ & $10(23,8)$ \\
\hline Total & $20(47,6)$ & - & $22(52,4)$ & $42(100,0)$ \\
\hline \multicolumn{5}{|l|}{ Vínculo Privado } \\
\hline Prestação de serviços & - & $7(46,7)$ & $9(40,9)$ & $16(43,2)$ \\
\hline Participação nos lucros & - & $4(26,7)$ & $9(40,9)$ & $13(35,1)$ \\
\hline Autônomo & - & $4(26,7)$ & $4(18,2)$ & $8(21,6)$ \\
\hline Total & & $15(40,5)$ & $22(59,5)$ & $37(100,0)$ \\
\hline \multicolumn{5}{|c|}{ Carga horária semanal (horas)* } \\
\hline$\leq 20$ & $4(20,0)$ & $4(26,7)$ & $0(0,0)$ & $8(14,0)$ \\
\hline $21-30$ & $2(10,0)$ & $2(13,3)$ & $5(22,7)$ & $9(15,8)$ \\
\hline $31-40$ & $13(65,0)$ & $6(40,0)$ & $7(31,8)$ & $26(45,6)$ \\
\hline$>40$ & $0(0,0)$ & $3(20,0)$ & $10(45,5)$ & $13(22,8)$ \\
\hline Não respondeu & $1(5,0)$ & $0(0,0)$ & $0(0,0)$ & $1(1,8)$ \\
\hline \multicolumn{5}{|c|}{ Renda mensal (salários mínimos)* } \\
\hline$>3$ & $7(35,0)$ & $8(53,3)$ & $1(4,5)$ & $16(28,1)$ \\
\hline $3-5$ & $11(55,0)$ & $6(40,0)$ & $19(86,4)$ & $36(63,2)$ \\
\hline$>5$ & $1(5,0)$ & $1(6,7)$ & $2(9,1)$ & $4(7,0)$ \\
\hline Não respondido & $1(5,0)$ & $0(0,0)$ & $0(0,0)$ & $1(1,8)$ \\
\hline \multicolumn{5}{|c|}{ Remuneração ideal (salários mínimos)* } \\
\hline $3-5$ & $7(35,0)$ & $5(33,3)$ & $0(0,0)$ & $12(21,1)$ \\
\hline $6-10$ & $10(50,0)$ & $9(60,0)$ & $16(72,7)$ & $35(61,4)$ \\
\hline $11-15$ & $1(5,0)$ & $1(6,7)$ & $4(18,2)$ & $6(10,5)$ \\
\hline $16-20$ & $1(5,0)$ & $0(0,0)$ & $2(9,1)$ & $3(5,3)$ \\
\hline Não respondido & $1(5,0)$ & $0(0,0)$ & $0(0,0)$ & $1(1,8)$ \\
\hline \multicolumn{5}{|l|}{ Classificação do salário* } \\
\hline Ótimo & $2(10,0)$ & $0(0,0)$ & $1(4,5)$ & $3(5,3)$ \\
\hline Bom & $5(25,0)$ & $4(26,7)$ & $9(40,9)$ & $18(31,6)$ \\
\hline Regular & $5(25,0)$ & $10(66,7)$ & $11(50,0)$ & $26(45,6)$ \\
\hline Ruim & $7(35,0)$ & $1(6,7)$ & $1(4,5)$ & $9(15,8)$ \\
\hline Péssimo & $1(5,0)$ & $0(0,0)$ & $0(0,0)$ & $1(1,8)$ \\
\hline Total & $20(35,1)$ & $15(26,2)$ & $22(38,6)$ & $57(\mathbf{1 0 0 , 0})$ \\
\hline
\end{tabular}

$*_{\mathrm{n}}=57$ que correspondem aos cirurgiões-dentistas que exercem atualmente a profissão e foram considerados os percentuais válidos 
Na tabela 4, estão detalhados os dados sobre a percepção do mercado, satisfação com a profissão e desafios enfrentados. A respeito da percepção do mercado de trabalho, a maior parcela considerou como regular (40\%). Em relação à satisfação com a profissão, apenas $11,4 \%$ relataram não estarem satisfeitos com a Odontologia (tabela 4). Os 67 participantes que já se inseriram no mercado $(95,7 \%)$ foram questionados quanto às dificuldades vivenciadas no início do exercício profissional. Todas as dificuldades foram relatadas por parte considerável dos egressos; as mais citadas foram baixa remuneração $(82,1 \%)$ e insegurança na prática clínica $(70,1 \%)$.

Tabela 4. Percepção dos cirurgiões-dentistas sobre o mercado de trabalho em Odontologia, satisfação com a profissão e dificuldades relatadas no início do exercício profissional

\begin{tabular}{|c|c|c|}
\hline $\begin{array}{l}\text { Variável } \\
\end{array}$ & $\mathbf{n}$ & $\%$ \\
\hline \multicolumn{3}{|l|}{ Percepção sobre o mercado } \\
\hline Ótimo & 0 & 0,0 \\
\hline Bom & 17 & 24,3 \\
\hline Regular & 28 & 40,0 \\
\hline Ruim & 19 & 27,1 \\
\hline Péssimo & 6 & 8,6 \\
\hline \multicolumn{3}{|l|}{ Satisfação com a profissão } \\
\hline Muito satisfeito & 8 & 11,4 \\
\hline Satisfeito & 36 & 51,4 \\
\hline Nem satisfeito, nem insatisfeito & 18 & 25,7 \\
\hline Insatisfeito & 7 & 10,0 \\
\hline Muito insatisfeito & 1 & 1,4 \\
\hline \multicolumn{3}{|c|}{ Dificuldades no início do exercício profissional* } \\
\hline \multicolumn{3}{|c|}{ Insegurança na prática clínica } \\
\hline Sim & 47 & 70,1 \\
\hline Não & 20 & 29,9 \\
\hline \multicolumn{3}{|c|}{ Dificuldade para encontrar o primeiro emprego } \\
\hline $\operatorname{Sim}$ & 28 & 41,8 \\
\hline Não & 39 & 58,2 \\
\hline \multicolumn{3}{|l|}{ Baixa remuneração } \\
\hline Sim & 55 & 82,1 \\
\hline Não & 12 & 17,9 \\
\hline \multicolumn{3}{|l|}{ Condições de trabalho inadequadas } \\
\hline $\operatorname{Sim}$ & 35 & 52,2 \\
\hline Não & 32 & 47,8 \\
\hline \multicolumn{3}{|l|}{ Dificuldade na captação de pacientes } \\
\hline Sim & 27 & 40,3 \\
\hline Não & 40 & 59,7 \\
\hline \multicolumn{3}{|l|}{ Falta de experiência administrativa } \\
\hline Sim & 36 & 53,7 \\
\hline Não & 31 & 46,3 \\
\hline
\end{tabular}

* n=67 que correspondem aos cirurgiões-dentistas que atuam no mercado ou já se inseriram, porém não exercem a profissão atualmente e foram considerados os percentuais válidos 
Como sugestões para o curso de Odontologia da UFPI, os egressos apontaram principalmente a abordagem de Administração, Marketing e Empreendedorismo (55,7\%), aprofundamento em Cirurgia $(17,1 \%)$ e a implementação de outras disciplinas $(14,3 \%)$, como Pacientes Especiais e Psicologia para Odontologia.

\section{DISCUSSÃO}

No presente estudo, a maioria dos participantes era do sexo feminino, com até 25 anos. A presença feminina na Odontologia tem se fortalecido desde a década de 80 , e atualmente $56,3 \%$ dos CD no Brasil são mulheres ${ }^{2}$.

Observou-se que o anseio dos egressos na busca pela pós-graduação é uma característica que permanece, corroborando outros estudos ${ }^{1,21-24}$. Os primeiros anos de exercício da profissão são focados na consolidação no mercado de trabalho e na especialização ${ }^{5}$. A educação continuada é vista como fator de qualificação e diferenciação, uma estratégia de competição no mercado de trabalho ${ }^{22,23,25}$, ou como uma forma de superar deficiências da graduação ${ }^{24}$. Relaciona-se ainda a predominância do tipo lato sensu à preferência pelas práticas clínicas tradicionais ${ }^{1}$. Embora haja esta procura por pós-graduação lato sensu, a UFPI oferece apenas dois cursos nessa modalidade para Odontologia, especialização em Ortodontia e residência em Cirurgia e Traumatologia BucoMaxilo-Facial. Neste estudo, as áreas mais citadas pelos entrevistados foram Cirurgia (principalmente em aperfeiçoamento), Endodontia e Ortodontia. As duas últimas são as áreas com maior número de $\mathrm{CD}$ especialistas no Brasil $^{16}$.

Apesar da saturação sugerida pelos números crescentes de cursos e profissionais ${ }^{17}$, a maioria dos egressos conseguiu se inserir no mercado em até 3 meses, assim como observado em outros trabalhos ${ }^{1,22}$. O fato é associado ao surgimento de novos postos de trabalho, tanto no serviço público, pela expansão da saúde bucal no setor, quanto no mercado privado, pelo incremento de clínicas populares, convênios e planos de saúde 1,22,23,26.

A distribuição da força de trabalho em Odontologia no Brasil é marcada pelo desequilíbrio $^{17}$, com concentração de profissionais nos centros urbanos $^{2,27}$. Nos resultados do presente estudo, observou-se que os $\mathrm{CD}$ se estabeleceram de forma equivalente entre a RMT e o interior, em concordância com um estudo do Piauí ${ }^{21}$, no qual $60 \%$ atuam no interior do estado. Supõe-se que essa melhor distribuição possa estar associada às oportunidades de emprego nas regiões do interior, que apresentam menor número de profissionais e maior demanda em saúde bucal, em contraste com a saturação de CD na capital ${ }^{17}$.

Em relação aos setores de atuação, observou-se que os CD estão inseridos de forma equiparável entre os cenários público e privado, porém com redução da prática exclusiva em consultório particular e predominância do exercício em ambos os setores. Dentro do setor privado, prevaleceram ainda as formas de atuação terceirizada sobre a autônoma. Tal resultado está de acordo com o observado em um estudo com egressos recentes do Ceará ${ }^{22}$, porém contrasta com outros, realizados no Rio Grande do Norte ${ }^{1}$, Tocantins $^{23}$ e São Paulo ${ }^{25}$, nos quais predominou a atuação privada. No entanto, o resultado confirma a tendência de redução progressiva da prática autônoma, em paralelo à ampliação do serviço público e das práticas da Odontologia de grupo $^{1,19}$. O assalariamento, antes visto com ressalvas, ganha a perspectiva da estabilidade financeira almejada, frente às incertezas do exercício autônomo ${ }^{15}$.

A absorção de profissionais no mercado privado é em parte devida à ascensão da saúde suplementar ${ }^{1,26}$. Todavia, tal segmento por vezes prioriza os ideais de mercado em detrimento da autonomia profissional e das condições de 
trabalho ${ }^{5,19}$. No setor público, chama a atenção a quantidade de profissionais que trabalham sem contrato, denotando a precarização de vínculos trabalhistas.

A maioria dos $\mathrm{CD}$ inseridos no mercado possuía renda mensal de até 5 salários mínimos, média semelhante à de um trabalho com egressos recentes $^{22}$ e inferior à de outros estudos que abordam CD com maior tempo de exercício da profissão ${ }^{1,21,23,25}$, nos quais a faixa predominante é de até 10 salários mínimos. Em relação à satisfação com o salário, a maior parte considerou sua renda como boa ou regular, e apontou como remuneração ideal almejada a faixa entre 6 e 10 salários mínimos, média coerente com a realidade brasileira ${ }^{1,2,21,23,25}$.

Os participantes julgaram o mercado de trabalho principalmente como regular, visão mais retraída do que o observado em outros trabalhos ${ }^{1,22}$, nos quais $40 \%^{1}$ e $41,4 \%^{22}$ apontaram boas perspectivas para a profissão. No entanto, em relação à satisfação com a escolha da profissão, apenas uma pequena parcela declarou-se insatisfeita.

Durante a transição da universidade para a vida profissional, os recém-formados se deparam com dificuldades no ingresso ao mercado de trabalho ${ }^{28}$. As dificuldades citadas pelos $\mathrm{CD}$, ainda que de certa forma esperadas no início do exercício profissional, podem sinalizar a situação desfavorável de um mercado saturado, assim como refletir, em parte, um distanciamento entre a vida acadêmica e a realidade ${ }^{29}$. Observa-se ainda que apesar da maioria ter ingressado no mercado em até 3 meses, 41,8\% relataram dificuldade para encontrar o primeiro emprego.

Como sugestão para o curso de graduação, os egressos apontaram o aumento da carga horária dedicada a noções sobre Administração, Marketing e Empreendedorismo nas disciplinas já existentes, ou mesmo a criação de um novo componente curricular do curso de Odontologia da UFPI, que poderia contribuir para a adaptação dos recém-formados ao mercado de trabalho, fato também observado por outros autores ${ }^{21,24,25,29}$. Tal necessidade surge uma vez que a Odontologia possibilita o trabalho em diversas modalidades, e o cenário autônomo competitivo exige tais atribuições dos $\mathrm{CD}^{24,29}$. Ainda há desafios a serem superados na aproximação entre a formação dos cirurgiões-dentistas e a realidade, especialmente para melhor preparar os egressos frente as adversidades vivenciadas no período de transição da universidade para o mundo do trabalho.

Aponta-se como limitações do presente estudo a abrangência da amostra, referente a apenas uma instituição de ensino, e a abordagem mais resumida sobre as dificuldades enfrentadas pelos cirurgiões-dentistas recém-formados, que merece uma discussão mais aprofundada.

\section{CONCLUSÕES}

O perfil dos egressos de 2017 e 2018 do curso de Odontologia da UFPI é predominantemente jovem, do sexo feminino, e que valoriza a pós-graduação. $\mathrm{O}$ mercado proporcionou oportunidades para uma inserção em tempo hábil, com renda mensal satisfatória, no entanto, com redução do exercício estritamente autônomo e maior tendência ao assalariamento. De uma forma geral, apesar das dificuldades, os egressos estão satisfeitos com a profissão.

\section{ABSTRACT \\ Insertion of graduates of a Dentistry course in Piaui in the job market}

Existent transformations and complexities within dental labor market raise doubts about dentists' inclusion in professional practice, and their training. This research, therefore, aimed to evaluate the entry of Federal University of Piauí dentistry course graduates into the labor-market. This cross-sectional, quantitative study, conducted by sending an online autoquestionnaire to 76 dentists, graduated between 2017 and 2018, addressing sociodemographic 
data, postgraduate studies and entry into the labor market, receiving response-rate of $92.1 \%$. Descriptive data analysis performed showed the majority of graduates $(88.6 \%)$ had or were taking a postgraduate course, mainly in Surgery, Endodontics and Orthodontics. Relative to market entry, $4.3 \%$ had never practiced the profession, and $75.7 \%$ entered within 3 months after graduation. Dentists were equally divided between public and private sectors, primarily working 40 hours/week $(75.4 \%)$, earning monthly income of up to 5 minimum salaries $(91.3 \%)$. Only $11.4 \%$ were not satisfied with the chosen profession, and $40 \%$ judged the level of market difficulty was intermediate. Among the difficulties reported at the beginning of the profession, the majority of dentists cited the low salaries $(82.1 \%)$ and insecurity in clinical practice $(70.1 \%)$. As a suggestion for the undergraduate course, they indicated that they could focus on Administration, Marketing and Entrepreneurship. The market was observed to provide opportunities for rapid inclusion, however with a reduction in exercising the profession in a strictly independent manner. Graduates placed value on postgraduate studies, and despite difficulties, they were satisfied with the profession.

Descriptors: Job Market. Dentistry. Dental Staff.

\section{REFERÊNCIAS}

1. Pinheiro IAG, Noro LRA. Egressos de Odontologia: o sonho da profissão liberal confrontado com a realidade da saúde bucal. Rev ABENO. 2016;16(1):13-24.

2. Morita MC, Haddad AE, Araújo ME. Perfil Atual e Tendências do Cirurgião Dentista Brasileiro. Maringá: Dental Press International, 2010.

3. Lucena EHG, Pucca Júnior GA, Sousa MF. A Política Nacional de Saúde Bucal no Brasil no contexto do Sistema Único de Saúde. Tempus (Brasília). 2011;5(3):53-63.

4. Cardoso AL, Vieira ALS, Machado MH.
Mercado de trabalho dos odontólogos no Brasil. Divulg saúde debate. 2010;(45):71-9.

5. Freitas CHSM. Dilemas no exercício profissional da Odontologia: a autonomia em questão. Interface Comun Saúde Educ. 2007;11(21):25-38.

6. Brasil. Portaria $\mathrm{n}^{\circ} 1.444$, de 28 de dezembro de 2000. Estabelece incentivo financeiro para reorganização da saúde bucal prestada nos municípios por meio do Programa Saúde da Família. Diário Oficial da União 29 dez 2000;Seção 1.

7. Moretti-Pires RO, Bueno SMV. Freire e formação para o Sistema Único de Saúde: o enfermeiro, o médico e o odontólogo. Acta Paul Enferm. 2009;22(4)439-44.

8. Brasil. Ministério da Saúde. Secretaria de Atenção à Saúde. Departamento de Atenção Básica. Coordenação Nacional de Saúde Bucal. Diretrizes da Política Nacional de Saúde Bucal. Brasília; 2004.

9. Moura MS, Ferro FEFD, Cunha NL, Sousa Nétto OB, Lima MDM, Moura LFAD. Saúde bucal na Estratégia de Saúde da Família em um colegiado gestor regional do estado do Piauí. Ciênc Saúde Colet. 2013;18(2):471-80.

10. Brasil. Ministério da Saúde. Secretaria de Atenção à Saúde. Departamento de Atenção Básica. Cobertura da Saúde Bucal set 2019. e-Gestor Atenção Básica. [Acesso em 24 nov 2019]. Disponível em: https://egestorab.saude.gov.br/paginas/acess oPublico/relatorios/relHistoricoCoberturaS B.xhtml.

11. Silveira JLGC, Garcia VL. Mudança curricular em Odontologia: significados a partir dos sujeitos da aprendizagem. Interface (Botucatu). 2015;19(52):145-58.

12. Brasil. Ministério da Educação. Conselho Nacional de Educação. Câmara de Educação Superior. Resolução CNE/CES 3, de 19 de 
fevereiro de 2002. Institui Diretrizes Curriculares Nacionais do Curso de Graduação em Odontologia. Diário Oficial da União 4 mar 2002;Seção 1.

13. Instituto Nacional de Estudos e Pesquisas Educacionais Anísio Teixeira. Sinopse Estatística da Educação Superior 2015. Brasília: Inep, 2016. Acesso em 21 jun 2019]. Disponível em: http://portal.inep.gov.br/basicacenso-escolar-sinopse-sinopse.

14. Instituto Nacional de Estudos e Pesquisas Educacionais Anísio Teixeira. Sinopse Estatística da Educação Superior 2018. Brasília: Inep, 2019. [Acesso em 10 nov 2019]. Disponível em: http://portal.inep.gov.br/basicacenso-escolar-sinopse-sinopse.

15. Cascaes AM, Dotto L, Bomfim RA. Tendências da força de trabalho de cirurgiões-dentistas no Brasil, no período de 2007 a 2014: estudo de séries temporais com dados do Cadastro Nacional de Estabelecimentos de Sáude. Epidemiol Serv Saúde. 2018;27(1):e201723615.

16. Conselho Federal de Odontologia. Estatísticas. 2020. [Acesso em 28 jul 28 2020]. Disponível em: http://website.cfo.org.br/estatisticas/.

17. San Martin AS, Chisini LA, Martelli S, Sartori LRM, Ramos EC, Demarco FF. Distribuição dos cursos de Odontologia e de cirurgiões-dentistas no Brasil: uma visão do mercado de trabalho. Rev ABENO. 2018;18(1):63-73.

18. Ferreira NP, Ferreira AP, Freire MCM. Mercado de trabalho na Odontologia: contextualização e perspectivas. Rev Odontol UNESP. 2013;42(4):304-9.

19. Moraes DA, Maluf F, Tauil PL, Portillo JAC. Precarização do trabalho odontológico na saúde suplementar: uma análise bioética. Ciênc Saúde Colet. 2019;24(3):705-14.

20. Nétto OBS, Moura MS, Lima MDM, Lages GP, Mendes RF, Moura LFAD. O Pró-Saúde no curso de Odontologia da Universidade Federal do Piauí (UFPI): relato de uma vivência de cinco anos. Cienc Cuid Saude. 2013;12(2):391-7.

21. Ferraz MAL, Nolêto MSC, Martins LLN, Bandeira SRL, Portela SGC, Pinto PHV, et al. Perfil dos egressos do curso de Odontologia da Universidade Estadual do Piauí. Rev ABENO. 2018;18(1):56-62.

22. Pinheiro VC, Menezes LMB, Aguiar ASW, Moura WVB, Almeida MEL, Pinheiro FMC. Inserção dos egressos do curso de odontologia no mercado de trabalho. Rev Gauch Odontol. 2011;59(2):277-83.

23. Costa BAO, Gonçalves CF, Zanin L, Flório FM. Inserção de egressos de Odontologia do Tocantins no mercado de trabalho. Rev ABENO. 2016;16(2):93-104.

24. Melo Júnior PC, Gurgel LGF, Gimarães RP, Beatrice LCS, Pedrosa MS, Silva CHV. Perfil dos egressos do Curso de Odontologia da Universidade Federal de Pernambuco. Rev ABENO. 2018;18(3):93-104.

25. Mialhe FL, Furuse R, Gonçalo CS. Perfil profissional de uma amostra de egressos da Faculdade de Odontologia de Piracicaba. UFES Rev Odontol. 2008;10(2):31-6.

26. Pietrobon L, Silva CM, Batista LRV, Caetano JC. Planos de assistência à saúde: interfaces entre o público e o privado no setor odontológico. Ciênc Saúde Colet. 2008;13(5):1589-99.

27. Gabardo MCL, Ditterich RG, Cubas MR, Moysés ST, Moysés SJ. Inequalities in the workforce distribution in the Brazilian Dentistry. Rev Gauch Odontol. 2017;65(1):70-6.

28. Teixeira MAP, Gomes WB. Estou me formando... e agora? reflexões e perspectivas de jovens formandos universitários. Rev Bras Orientac Prof. 2004; 5(1):47-62. 
29. Saliba NA, Moimaz SAS, Prado RL, Garbin

CAS. Percepção do cirurgião-dentista sobre formação profissional e dificuldades de inserção no mercado de trabalho. Rev Odontol UNESP. 2012;41(5):297-304.

\section{Correspondência para:}

Patrícia Ferreira de Sousa Viana

e-mail: patriciaviana@ufpi.edu.br

Campus Universitário Ministro Petrônio

Portella - bloco 5

Bairro Ininga

64049-550 Teresina/PI 